Rev. Asoc. Esp. NeuropsiQ. 2020; 40(I37): 73-9I

DOI: I0.432 I/SO2 I I-57352020000I00005

\title{
Conciencia y naturaleza: en los límites del fisicalismo
}

Consciousness and nature. The limits of physicalism

\section{Esther Benzaquén de Hevia}

Doctoranda UNED. Filosofía, especialidad Filosofía de la ciencia.

Correspondencia:esbenzaquen@gmail.com

Recibido: 24/06/2019; aceptado con modificaciones: 15/1/2020

Resumen: Con la esperanza de que los conocimientos en neurociencia ayudasen a comprender al ser humano y a tratar de modo científico sus pérdidas de equilibrio mental, durante los últimos años del siglo XX se dedicaron grandes esfuerzos a la investigación del cerebro, dando lugar a la comúnmente denominada psiquiatría biológica. Sin embargo, debido a los límites y conveniencia de estos conocimientos en su aplicación práctica, junto con las dudas que proporciona la ciencia actual sobre la percepción humana, un número creciente de científicos y filósofos han abierto nuevas líneas de investigación y desarrollado nuevas hipótesis sobre la actividad mental y las relaciones mente-materia que podrían cambiar nuestra comprensión del mundo.

Palabras clave: conciencia, mente, materia, objetividad, subjetividad.

Abstract: In the hope that knowledge in neuroscience helped to understand human beings and treat their losses of mental balance in a scientific way, during the last years of the 20th century great efforts were devoted to brain research, leading to the so-called "biological psychiatry". However, due to the limits and convenience of this knowledge in its practical application, together with the existing doubts regarding current science about human perception, an increasing number of scientists and philosophers have opened new lines of research and developed new hypotheses about mental activity and mind-matter relationships that might change our understanding of the world.

Key Words: consciousness, mind, matter, objectivity, subjectivity. 
Cundo la ciencia investiga un fenómeno, a menudo olvida que el método que utiliza procede de un modo de entender la naturaleza que es filosófico y cultural. El origen de la ciencia que conocemos (Descartes o Galileo) se basó en la separación de la mente y el cuerpo, considerando que solo podía ocuparse de la materia, a la que podía medir o cuantificar para así elaborar leyes, mientras que la mente inmaterial quedó a cargo de la religión y posteriormente de la psicología. En el momento en que la ciencia abordó la actividad mental, se consideraron dos opciones para resolver el dualismo: o bien la mente procedía del cerebro (se trata de un epifenómeno de la actividad cerebral), o bien es inaccesible desde la ciencia por ser inmaterial.

En la actualidad, el dualismo ha desaparecido casi por completo, al menos en el ámbito científico, pero el modo en que lo ha hecho, su tratamiento de la mente como un epifenómeno, tiene sus límites explicativos e inconvenientes prácticos. El problema más importante que presenta es su incapacidad para explicar cómo la materia se convierte en mente, que es el viejo problema materia-cuerpo y también un problema de causalidad. De este problema surgen, además, contradicciones en el aspecto práctico, como la negación del libre albedrío o capacidad de decisión de los humanos, características que constituyen la base de las sociedades democráticas.

El problema filosófico mente-cuerpo no es un tema banal para la psiquiatría, sino que se encuentra en el origen del actual modelo comúnmente denominado biologicista, centrado en el cerebro, cuyo principal escollo se encuentra en el abandono del estudio de las influencias sociales y culturales, excluyendo las relaciones humanas, principal ámbito en el que viven los seres humanos, bajo el presupuesto de que todo proviene de la materia y, por tanto, si un comportamiento es problemático, su origen se encuentra en el sustrato material. Carl Gustav Hempel (1905-1997) fue un filósofo de la ciencia, representante de las ideas dominantes del neopositivismo durante el siglo XX y un importante autor sobre epistemología que introdujo sus ideas sobre la taxonomía de los trastornos mentales en la American Psychopathological Association $(1,2)$, según las cuales la única forma de comprender los trastornos mentales era tratarlos desde la física aplicada. Aunque el método de Hempel está basado en leyes causales, en el caso de la psiquiatría, se evitó este aspecto suponiendo que dichas leyes se encontrarían más tarde, permaneciendo, en cambio, su método.

Para la perspectiva materialista (ontológicamente reduccionista), si la materia es estudiada, habrá una explicación completa: solo hay que averiguar las causas que producen los fenómenos. Sin embargo, todo lo que se ha encontrado en la relación mente-materia son correlaciones, sin poder establecer una causalidad directa. Además de no ofrecer una respuesta satisfactoria, de este modo se estaría incurriendo en un nuevo dualismo: materia como sustrato y mente como producto de la materia. 
Este modelo materialista reductivo aporta un problema adicional: no se tiene en cuenta el problema del observador, dando por hecho la perspectiva de tercera persona de la ciencia estándar. La relación sujeto-objeto fue resuelta por Immanuel Kant (3) y por la filosofía fenomenológica de Edmund Husserl (4) y Martin Heidegger (5), pero estos últimos son autores que, debido a sus críticas al positivismo, se apartaron de la ciencia de su época, con excepción de Maurice Merleau-Ponty, quien incluso trata la posibilidad de que la física cuántica explique el comportamiento humano: "Or, sans doute la physique quantique nous a appris à introduire dans notre image du monde physique des données 'acausales' (...) derrière lesquelles il n'y a pas lieu d'affirmer par principe une causalité du type classique, et les physiciens eux-mêmes (...) on fait la comparaison" (6). En este autor se han inspirado algunos investigadores actuales, como, por ejemplo, Varela (7) o Walter Freeman y Giuseppe Vitiello (8), para elaborar nuevas hipótesis y experimentos sobre el comportamiento humano. A pesar de las reticencias de muchos fenomenólogos tradicionales, la lista de autores interesados en la naturalización de la fenomenología (como método científico) es importante: Josef Parnas, Louis A. Sass y Dan Zahavi (9); Shaun Gallagher (10) o Thomas Fuchs (11), autores que proponen la colaboración y conciliación de los conocedores de la fenomenología con las nuevas investigaciones sobre la conciencia como solución al actual vacío científico de la psiquiatría.

En resumen, el problema de la relación mente-cuerpo sigue abierto y en busca de soluciones que den una respuesta satisfactoria. A medida que se han hecho progresos en la comprensión del cerebro, se ha ido descubriendo que se trata de uno de los sistemas conocidos más complejos, sobre todo, en la relación materia-mente, lo que ha llevado a estudiarlo también desde la física cuántica, con la seguridad de que, al igual que en todo el mundo material y en todos los sistemas biológicos, en el cerebro se producen eventos cuánticos. Son estudios controvertidos, pero son los únicos que ofrecen una explicación razonable dentro de la ciencia para la relación mente-materia. La hipótesis de David Bohm (12), aunque es la más sencilla de entender, va más allá de la física cuántica actual sin que se hayan llevado estudios experimentales con ella. Hay que tener en cuenta que se trata de un nuevo campo de estudio científico de gran dificultad, a pesar de lo cual el simple hecho de plantear que se trata de un problema no resuelto científicamente ya constituye un paso importante en su comprensión.

El objetivo de este artículo es reflexionar, fundamentalmente desde el punto de vista epistemológico, sobre las dificultades de un modelo derivado de un realismo ingenuo que intenta explicar la subjetividad a partir únicamente del funcionamiento cerebral. En la primera parte de este artículo, presento la teoría emergentista, según la cual la mente es un producto de la actividad neuronal; en la segunda, los problemas y falta de explicación de este presupuesto junto con su origen; y en la tercera, 
propongo una posible solución epistemológica dentro del marco de la física cuántica, que, en contraposición a las dificultades del mecanicismo tradicional, facilita la comprensión del problema materia-mente.

\section{LA MENTE EXPLICADA COMO UN EPIFENÓMENO}

Los límites para la comprensión de la mente están marcados por el mismo enfoque de la explicación. Es lo que se denomina reduccionismo materialista, que consiste en explicar la actividad mental mediante sus correlatos físicos con la convicción de que el cerebro produce la mente. Sin embargo, el conocimiento de los correlatos no significa que los mecanismos cerebrales sean la causa directa de la actividad mental. Es un problema muy bien explicado por David Hume (13): que dos fenómenos sucedan al mismo tiempo no significa que uno sea la causa del otro; incluso, por los motivos que se explican más adelante, puede pensarse lo contrario, que la actividad mental sea la causa de los cambios neuronales.

Ante el problema mente-cuerpo, existen diferentes actitudes: se puede afirmar que la mente no es un asunto científico, como afirma el cartesianismo; se puede decir que no es un problema que la ciencia actual pueda tratar; o se puede negar. Una postura radical y muy influyente en el problema mente-cuerpo es el eliminativismo materialista (14), que consiste en un monismo que niega la necesidad de discutir los estados mentales, disolviendo así el problema negándolo: la conciencia es algo así como una ilusión y, por tanto, no existe el problema mente-cuerpo. De este modo, mente y psicología quedan fuera de la ciencia, incluso las correlaciones mente-cerebro tendrían poco sentido.

\section{a) Se niega que exista un problema mente-cuerpo}

El más conocido partidario del eliminativismo es Daniel Dennett (15), cuyo argumento consiste en afirmar que el fenómeno de la conciencia es solo una capacidad para informar y discriminar (como, por ejemplo, el dolor que sirve para informar de que algo va mal), pero que no existe en ningún otro sentido. Este argumento parece ajustarse a una concepción materialista estricta: solamente existe lo que se puede medir o cuantificar. Según este presupuesto, la creencia en que existe la conciencia se debe a una ilusión o una cuestión fuera de la ciencia procedente del materialismo cartesiano, y se interpreta como un teatro en el cual un espectador interno ve mentalmente las experiencias. Dennett considera, por tanto, que es una cuestión social que se puede explicar a través de los denominados memes, elementos culturales procedentes de la evolución que producen dicha ilusión de conciencia, una especie de truco de la naturaleza para ayudar a la supervivencia. La cuestión es que, por una parte, este intento de solución por medio de la disolución del proble- 
ma no explica cómo se produce lo que denomina ilusión y, por otra, resulta circular, porque cualquier idea que se afirme pertenecerá a la ilusión que producen los memes, lo que incluye a la propia teoría eliminativista.

\section{b) La ilusión de la conciencia}

Una postura similar es defendida por Keith Frankish (16), un autor aún más radical que Dennett, quien califica de ilusionismo a los intentos de explicación científica de la subjetividad. Según este autor, se está sustituyendo el problema mente-cuerpo por dicho ilusionismo cuando lo que hay que investigar es el sustrato material, es decir, el cerebro. El argumento es similar al de Dennett: se apoya en la teoría evolutiva para afirmar que la naturaleza ha creado un poderoso mecanismo que hace que los seres humanos crean que tienen conciencia, pero solo se trata de una ilusión, un truco evolutivo con fines de supervivencia, por lo que los que defienden o investigan la conciencia son meros ilusionistas que refuerzan un engaño generalizado de la psicología popular. La insistencia en los mismos argumentos no ańade nada nuevo: de acuerdo en que hay que dar explicaciones razonables y dentro de la ciencia, lo que no se justifica es la negación de la subjetividad, porque no aportan ninguna prueba salvo su insistencia en permanecer en el marco conceptual de la neurociencia clásica.

El conocido psiquiatra Thomas Szasz (17) expuso el problema de forma muy clara con su metáfora del programa televisivo: sería como culpar a la falta de sintonización del televisor de la mala calidad de un programa. Indudablemente, es importante que el aparato de televisión (el cerebro) funcione correctamente, pero ello no mejorará el programa. El contenido (el programa de la metáfora de Szasz) es la información que circula entre el entorno y el individuo. Naturalmente, si no se dispone de un aparato de televisión (cerebro), no se puede ver el programa (no se puede percibir), pero eso no tiene que ver con la calidad del programa. Esta metáfora podría dar la razón a los que piensan que el cerebro es similar a una computadora (funcionalistas), pero la actividad mental es aún más complicada. Edelman y Tononi (18) explican cómo las experiencias modifican las redes neuronales, y desde las investigaciones cuánticas de la mente (19), se afirma que la mente actúa sobre la materia, sin olvidar la teoría enactivista de Francisco Varela (7), que considera que la mente no se encuentra solo en el cerebro, teoría que, en su versión más drástica (20), afirma que la conciencia es una propiedad fundamental de la naturaleza.

\section{Problemas del paradigma Clásico}

No es fácil explicar desde el punto de vista estrictamente materialista cómo el cerebro, con propiedades físicas, da lugar a la mente, cuyas propiedades son inmate- 
riales, por lo que se ha elaborado una complicada explicación de tipo emergentista denominada superveniencia (21), basada en el concepto de que el todo es mayor que la suma de las partes; por tanto, la mente, que es la suma de las partes, tendría propiedades que no posee el cerebro. Pero no hay ninguna demostración empírica de que sea así, sino que es solo una especulación basada en una forma de concebir la ciencia; es decir, analizando el funcionamiento de un mecanismo para establecer leyes que expliquen los efectos que se producen a partir de unas causas, suponiendo que las leyes físicas que se conocen sean completas. Desde un punto de vista más concreto, las investigaciones en neurociencia se han encontrado con el "problema difícil” (hard problem) (22), que es el problema semántico de cómo un significado ${ }^{1}$ se convierte en un proceso electro-químico en el cerebro, planteado por primera vez por John Searle con su conocida metáfora de la habitación china (23). El problema que contiene la metáfora de Szasz antes mencionada, el paso del significado a su codificación, aunque abundantemente discutido, marca los límites de la computación. En el caso de los humanos, por ejemplo, no puede explicar cómo una señal de cualquier tipo que avisa de un peligro se convierte en sustancias químicas en el cerebro que, a su vez, producen un estado de conciencia.

\section{a) Los qualia}

Pero aún hay más problemas cuando se intenta describir la mente como un epifenómeno. En la actividad mental hay que incluir las sensaciones, los denominados qualia (las cualidades subjetivas de las experiencias individuales). Los filósofos empiristas de la Ilustración, como John Locke (1632-1704), distinguieron entre cualidades primarias (objetivas) y cualidades secundarias (subjetivas), que son los actuales qualia, pero el positivismo del siglo XIX y su heredero del siglo XX, el positivismo lógico, en su búsqueda de legitimidad científica, redujeron todas las cosas a cualidades primarias, argumentando que el éxito de la ciencia se debe a que la mente percibe exactamente lo que hay en el mundo. A partir de ese momento, la actividad mental se convierte en un problema, un molesto fantasma en la máquina con el que no se puede hacer mucho desde la ciencia. Si ese es el planteamiento general de la ciencia del siglo XX, entonces es lógico que se afirme que la conciencia emerge de la materia.

Según esta forma de entender la actividad mental, el cuerpo-cerebro es solo una máquina que se relaciona con el medio ambiente a través de circuitos por los que recibe estímulos a los que responde y que puede averiarse como lo hace cualquier

${ }^{1}$ El término significado es entendido según la teoría de la información de Charles W. Morris (24) según la cual se distinguen las dimensiones sintáctica, semántica y pragmática. El significado pertenece a la semántica y se codifica en secuencias de signos (sintáctica) para convertirse en operacionalmente accesible (pragmática). 
máquina. La seguridad en esta concepción reduccionista de la mente-cerebro humana ha llevado al desarrollo de máquinas muy útiles para el trabajo y la información, pero no está clara la similitud entre estas máquinas y los seres humanos o biológicos en general (si aún no conocemos el funcionamiento del cerebro, difícilmente lo podremos imitar), lo que supone una muestra más de la limitación de las investigaciones reduccionistas y del modelo biológico y médico basado en estos presupuestos. De hecho, no podemos limitar la conciencia al cerebro, sino que numerosos estudios neurocientíficos (25-27) afirman que todo el cuerpo está implicado, lo que supone que también su entorno está involucrado, ya que el sistema nervioso se encuentra en comunicación permanente con el exterior.

\section{b) El dualismo mente-cuerpo}

El problema del dualismo no es sino una abstracción con el fin de poder controlar la naturaleza de una forma relativamente sencilla e intuitiva, pero se vuelve una noción problemática porque hay que dividir al organismo abstrayendo la mente de su cuerpo o al contrario, según la actividad del individuo. Un problema derivado de este modo de concebir la ciencia es su definición de naturalista, que implica, o bien un dualismo porque supone que existe algo que no es natural (lo artificial), o simplemente que se está en el lado de la verdad (una incuestionable autoridad científica), que es la ciencia dominante, con lo cual cualquier crítica queda automáticamente desprestigiada y definida como perteneciente al oscurantismo anterior a la aparición de la ciencia. No es de extrañar que, ante estos planteamientos científicos, hayan surgido filosofías irracionalistas que proponen acercarnos a nuestra verdadera realidad instintiva prescindiendo de un pensamiento más elaborado que nos alejaría del supuesto orden natural auténtico. La supresión del dualismo no debería suponer una renuncia a la racionalidad, sino todo lo contrario: se debería corregir lo que tiene de irracional el reduccionismo al pretender extender su método a cuestiones que ha excluido previamente, como es el caso de la mente (y sus creaciones).

\section{c) El problema del observador}

El principal problema que afronta el estudio de la conciencia es su propia naturaleza: la ciencia es por definición el estudio desde la tercera persona, por lo que, en este caso, el cerebro se estudiaría a sí mismo, lo que presenta la dificultad de que el método debería ser adaptado de forma que el experimento realizado en primera persona pueda ser incluido dentro de un proceso intersubjetivo en el cual se pudiera convertir en tercera persona (28). Sabemos, según las investigaciones en neurodinámica, que los cambios en el sistema nervioso se producen de modo paralelo 
a los cambios mentales, pero ignoramos las relaciones entre ambos aspectos, ya que desde el empirismo lógico no es posible establecer un puente que los una (binding problem). De modo que la sustancia tercera spinozista incognoscible es rechazada como una cosa en sí debido a que los humanos solo podemos procesar los fenómenos a través del esquema espacio-temporal kantiano (3). Herbert Feigl (28) propone que materia y mente sean considerados como dos lenguajes: los estados de conciencia privados pueden ser descritos en términos físicos neurológicos aunque solo podamos obtener datos del estado físico y ninguna información sobre el contenido cualitativo.

El resultado del convencimiento de que no se puede conocer la experiencia en primera persona es un rechazo frontal de la metafísica a partir de los siglos XIX-XX como una cuestión ajena a la ciencia, ya anticipada por Kant (3) con su teoría de la imposibilidad de conocer los noúmenos. Por eso, el neopositivismo invita incluso a no hablar de ellas (29). Sin embargo, las extrańas leyes de la mecánica cuántica (dualidad onda-partícula, entrelazamiento y problema de la medición) han hecho que se replantee el problema de lo que consideramos real (30) y hasta qué punto dicha realidad es construida por nuestra subjetividad, temas que son debatidos actualmente incluso desde la filosofía analítica $(22,31,32)$, disciplina habitualmente considerada la representante del neopositivismo, así como por la filosofía de la deconstrucción postmoderna, que siempre sospechó de la ciencia oficial (33). Se trataría de conceptualizar aquello que está oculto a simple vista (34), de lo que hasta ahora hemos considerado trascendente (o inexistente, según algunos), imposible de conocer, en contraste con lo inmanente, que sí podemos conocer y manipular.

\section{INTENTO DE RESOLUCIÓN DEL PROBLEMA MENTE-CUERPO}

En la Modernidad triunfó el dualismo cartesiano, pero también surgió un monismo: el monismo de Baruch Spinoza y su posterior interpretación por parte de Bertrand Russell, que constituyen la base de las investigaciones del problema mente-cuerpo bajo los principios de la teoría cuántica.

\section{a) El monismo de Spinoza}

Baruch Spinoza (35) fue el primero en tratar científicamente los aspectos de la naturaleza, el alma y Dios de distinta forma, no estrictamente como materia, aunque habitualmente ha sido interpretado en el sentido de que todo lo que existe puede reducirse a materia, adaptando su pensamiento a la concepción dominante de la ciencia (36). La conocida declaración de Spinoza de que "la extensión es un atributo de Dios, o, dicho de otro modo, Dios es cosa extensa" (35, p.186) es, en general, citada como una declaración de materialismo extremo. Sin embargo, Spinoza 
propone un monismo en el que lo mental y lo psíquico son una misma sustancia, no distintas cosas: los cuerpos son parte del todo y la mente es la idea de ese todo: "El pensamiento es un atributo de Dios, o, dicho de otro modo, Dios es cosa pensante", (35, p. 80). Pero no habla de un todo sin idea (o significado) como se propone desde el materialismo, sino que parece todo lo contrario. Si entendemos por Dios la realidad última que parece estar en el pensamiento de Spinoza, el pensamiento sería el atributo más importante de la naturaleza.

Aunque eliminemos de ese pensamiento el exagerado racionalismo de su época, Spinoza está afirmando que en ese todo de la naturaleza hay algo más que materia. Incluso su concepción panteísta de la naturaleza puede ser interpretada en el sentido de incluir el aspecto psicológico de las cosas. Como extensión y pensamiento son dos sistemas (o atributos) independientes, no tienen relación causal, pero sí una correlación: por cada modo de extensión hay un correspondiente modo de pensamiento. En relación al ser humano o cosa compleja, Spinoza afirma que todo lo que sucede en el cuerpo se refleja en la mente, pero que esta no interactúa con el cuerpo. Para Spinoza, la sustancia que produce estos dos aspectos o atributos es incognoscible. Asimismo, actualmente, no es posible investigar mente y cerebro como una misma sustancia, ya que, aunque tengan un sustrato común, mente y materia tienen desarrollos diferentes, pero tampoco es necesario discutir la palabra incognoscible, término que corresponde a la física de la época o al cuestionable determinismo spinozista.

\section{b) El monismo neutral}

En el siglo XIX, Bertrand Russell resuelve la contradicción del paralelismo spinozista con la creación de su teoría de un monismo neutral (equivalente a la sustancia incognoscible de Spinoza), en el que los contenidos de la mente y las cosas existentes en el exterior no son cosas distintas, sino aspectos de la misma sustancia, eliminando así cualquier posibilidad de complicada explicación emergentista con una solución que pretende resolver al mismo tiempo los problemas científicos de la inducción y los de mente-cuerpo. En el monismo neutral russelliano, al contrario que en el evolucionismo tradicional, la mente no es una consecuencia de los procesos cerebrales, sino que forma parte de ellos, de la física, y solo depende de su ordenación. La consecuencia es que todos, organismos vivos y no vivos, están constituidos por los mismos elementos de la naturaleza, organizados de diferente forma.

\section{c) Paralelismo y correlatos de la conciencia}

Un aspecto importante en el problema mente-cuerpo es distinguir entre descripción y explicación. Por ejemplo, los correlatos neuronales describirían el aspecto 
físico de la subjetividad, pero no aportan ninguna explicación de cómo se producen, porque, para obtener una explicación según la ciencia, es necesario demostrar que una causa produce un efecto.

La Teoría de la Información Integrada (IIT) (37) investiga directamente los correlatos de la conciencia. Aunque la IIT no pretende explicar el problema de cómo surge la conciencia (no se encuentra dentro del ámbito de la teoría cuántica), considera que podría explicar los trastornos mentales por el grado de integración de los elementos de la conciencia, lo que explicaría parte de la actividad mental, la consciente. El problema es que deja fuera de la explicación al paso del inconsciente al consciente. En cambio, otras investigaciones $(19,38)$, basadas en el monismo neutral, que se detallan más adelante (en el apartado La aplicación de los conceptos cuánticos a los sistemas mentales), proponen la unidad de un campo óntico subyacente no epistemológicamente accesible del que emergerían los aspectos mente-materia, así como una explicación del paso del inconsciente al consciente.

Las relaciones entre estados mentales y materiales pueden ser entendidas de forma no reductiva. Niels Bohr (39) tuvo la idea de que las características de la teoría cuántica, como la complementariedad onda-partícula, tenían también importancia fuera del campo de la física. De hecho, la noción de complementariedad procede de William James (40). Aunque Bohr estaba convencido de esta complementariedad, nunca concretó esta idea en programas de investigación, que apenas acaban de comenzar en la actualidad (19), capaces de aplicar la teoría cuántica más allá de la física aunque están en su inicio. El punto de partida es el cambio de dirección causal: no solo de materia a mente, sino también de mente a materia y de una nueva tercera categoría materia-mente neutral subyacente, en la que no se separan los dos aspectos -materia y mente-, sino que son consideradas emergentes de esta tercera categoría neutral (19), de modo muy parecido a como la concibieron Spinoza o Russell.

\section{d) El orden implicado}

El físico y también filósofo David Bohm (1917-1992) intentó solucionar el problema de la naturaleza de la mente con una propuesta sobre la arquitectura fundamental de la materia, la naturaleza de la mente y de cómo se relaciona con la materia, e incluso una propuesta sobre cómo la conciencia percibe el tiempo $(41,42)$. Su teoría, por tanto, puede ayudar a clarificar estos problemas. La idea de Bohm de que todo el universo envuelve a cada parte resulta extrańa y casi poética, muy cercana a la idea monadológica de Leibniz, y, sobre todo, contraintuitiva, pero puede entenderse en términos de información: en cualquier pequeña región del espacio hay un movimiento de ondas electromagnéticas que transporta la información del mundo entero; por ejemplo, una experiencia visual (ondas de luz) es después revelada (o 
descodificada) por los ojos y por un complejo proceso del cerebro, el cual también aporta información; el resultado es una experiencia visual del mundo en tres dimensiones. Para Bohm, el universo no es un conjunto de elementos separados entre sí, sino un todo indivisible interconectado por principios cuánticos de interconexión y simultaneidad entre las partículas.

Según la teoría del orden implicado (41), la mecánica clásica constituye una abstracción de la fundamental descripción de la naturaleza de la mecánica cuántica (la mecánica clásica sería la apariencia y la mecánica cuántica, la realidad platónica). De modo que las ventajas de la explicación cuántica de la subjetividad serían varias: desde la ciencia, por la simplicidad de su explicación y desde la perspectiva de nuestra comprensión del ser humano y de la propia naturaleza en general, por la incorporación de los estados de conciencia, del movimiento y del cambio continuo. En definitiva, la aplicación de esta teoría supondría la desmaquinización o humanización como alternativa al materialismo reductivo de la biología y la medicina, sobre todo, en sus especialidades psicológica y psiquiátrica. El mundo no sería un gran mecanismo, sino algo más parecido a una gran mente.

Al contrario de lo que sucede en mecánica clásica, todo el espacio está lleno de esa información que proporciona una conexión directa entre todos los sistemas, de forma que el todo es el responsable de la organización de las partes, aunque eso no impide cierta independencia de las partes. Las conexiones entre las partes de este orden implicado, que es indivisible, no tienen nada que ver con el espacio o el tiempo, sino con una cualidad denominada envolvimiento, y el orden explicado es la manifestación de una energía más profunda (el orden implicado) que Bohm y Hiley describen con la metáfora de un holograma (42) como forma de percepción humana. Igualmente, tras sus numerosos experimentos, Karl Pribram (43) afirmó que la percepción no consiste en que la memoria almacene partes distintas y después las reúna o relacione, sino en algo similar a un holograma: se trata de múltiples imágenes completas que dependen de muchas partes del cerebro.

En la teoría cuántica, también se suele reconocer que el proceso de medición implica cierto grado de libertad para elegir qué medir e influir en lo que se mide, y esto se ve solo como una instancia particular en las elecciones. El futuro es hasta cierto punto abierto, y al elegir qué medir, los sistemas están participando e influyendo en las posibilidades que se producirán. Si la biosfera y el cosmos son creativos de esta manera, la fe de los biólogos en que, en última instancia, las explicaciones que reducen los organismos a la química y la física finalmente tendrán éxito está completamente fuera de lugar. No hay ninguna razón en absoluto para suponer este esquema; por el contrario, hay muchas razones para otorgar el reconocimiento apropiado de las características únicas de la vida que han surgido en el devenir creativo de la naturaleza y para reconocer que es esencial reconocer la apertura de este devenir. No 
solo es necesario entender que cada organismo, incluidos los protozoos y las plantas, tienen mundos con un significado para ellos al que responden en consecuencia, sino que es también necesario reconocer que estos mundos están abiertos en cierta medida y que, para sobrevivir, los organismos tienen que enfrentar y lidiar con esta apertura. Al hacerlo, pueden darse cuenta de nuevas posibilidades que nunca antes se habían realizado o incluso se habían previsto, cuestión que es evidente en la observación y la percepción de que la evolución depende de la actividad de exploración inquieta de los animales, que descubren nuevos usos de lo que tienen disponible en su lucha por la vida.

\section{e) La concepción de la materia}

De acuerdo con la ampliamente aceptada teoría cuántica de campos para explicar la materia, las partículas son entendidas como la actividad subyacente de los campos, concepción muy alejada de nuestra percepción ordinaria. Es el movimiento del campo el que contiene información acerca de todo el universo; por ejemplo, la información de la luna existe en todo el espacio de la Tierra y por eso podemos percibirla. De forma análoga, cada partícula del cuerpo contiene información de todo el universo. Si trasladamos estos conceptos a la existencia humana, quiere decir que cada uno de nosotros forma parte del mundo y recibe/envía información, y que cualquier cambio en el entorno, por pequeño que sea, puede tener un profundo efecto. Incluso recientes experimentos del llamado EPR (la paradoja de Albert Einstein, Boris Podolsky y Nathan Rosen sobre el misterio del entrelazamiento cuántico), realizados por Bohm junto a John Bell (44), mostraron que cualquier cambio en el estado de un fotón (medición o polarización) puede tener un efecto instantáneo en el comportamiento de otro fotón situado hasta a 50 kilómetros. Por tanto, existe cierta clase de fuerza implicada en el comportamiento de la materia del todo. Sin embargo, la teoría de Bohm no niega que existan relaciones externas entre las partes (las leyes newtonianas), sino que estas se producen en el orden explicado y son las más comunes en nuestra experiencia; es decir, que en el universo hay algo más que el mecanismo del orden explicado en el que las piezas son como las de un lego: aunque percibimos cada parte independientemente una de otra, todas están relacionadas entre sí.

En la teoría de Bohm $(41,42)$, la información es entendida como el efecto del potencial que da forma al campo que actúa sobre la partícula; es decir, que el campo contiene información que literalmente informa o da forma a la partícula, lo que da lugar al concepto de información activa. La información sería el puente entre los aspectos mental y físico que se mueve en las dos direcciones a través de los distintos niveles que Bohm propone. Aunque, de las dos direcciones, el sentido de la mente hacia la materia sea el más difícil de entender, puede ser explicado mediante la información activa. 


\section{f) La aplicación de los conceptos cuánticos a los sistemas mentales}

También los conceptos de la mecánica cuántica pueden utilizarse para referirse a la mente sin tratar del cerebro. Según Atmanspacher (19), es posible que la actividad mental sea cuántica y esté correlacionada con una actividad cerebral completamente clásica. Dicha actividad mental cuántica es semejante a la lógica no booleana, lo que significa que las proposiciones pueden tener más precisión que los valores de la lógica binaria de verdad o falsedad; por ejemplo, pueden tener credibilidad o plausibilidad, lo que es mucho más parecido a la mente en la vida cotidiana. Este comportamiento de la mente es denominado por Atmanspacher como no conmutativo.

Con el fin de proporcionar un marco formal a la psicología en algunas de sus aplicaciones concretas sin reducirlas a sus correlatos neuronales, Atmanspacher (19) propone generalizar la teoría cuántica más allá de la física. Algunas de estas aplicaciones son: los procesos de decisión, la percepción biestable, la agencia cuántica y las correlaciones cerebro-mente.

\section{* Los procesos de decisión}

La clave es definir las probabilidades para los resultados de decisión y los tiempos de decisión en términos de amplitudes de probabilidad cuántica. Busemeyer et al. (45) encontraron acuerdo con un modelo espacial de Hilbert adecuado con datos empíricos, pudiendo aclarar los efectos de conjunción y disyunción en la toma de decisiones.

\section{* La percepción biestable}

La percepción de un estímulo es biestable si el estímulo es ambiguo, como el cubo de Necker. Se trata de una predicción teórica basada en el efecto Zeno ${ }^{2}$ (46): los sistemas mentales no están localizados bruscamente a lo largo del eje del tiempo, sino que parecen extenderse durante un intervalo de tiempo extendido en el que no se puede designar lo anterior o lo posterior.

\section{* El proceso de aprendizaje}

En estudios teóricos (47) se demuestra que, en tareas de aprendizaje simples, las pequeñas redes recurrentes no solo aprenden la relación de entrada-salida, sino también la secuencia en la que se han presentado las entradas.

\footnotetext{
${ }^{2}$ En mecánica cuántica, la paradoja Zeno o efecto Zeno consiste básicamente en que el observador interviene en el comportamiento de la materia (los átomos); es decir, se trata de un proceso causal entre las acciones y potencialidades cuánticas y los estados neuronales altamente organizados: lo observado está fuertemente ligado al ambiente (observador) y no pueden considerarse independientes uno del otro.
} 


\section{* La agencia según la teoría cuántica}

La idea es cómo los agentes pueden desarrollar su agencia como una especie de independencia de su entorno y las leyes deterministas que lo rigen (48), simulando el comportamiento del agente como probable cuántico no determinista en su espacio de memoria.

\section{${ }^{*}$ Las correlaciones cerebro-mente}

El punto de partida es un enfoque de doble aspecto de una realidad subyacente. En este marco conceptual, para lograr el acceso epistémico (no óntico), se distingue mente de materia como una herramienta básica. En consecuencia, el estado del campo psicofísico neutro subyacente es considerado óntico en relación con la distinción mente-materia, que es epistémica, por lo que ambos no son reducibles al campo subyacente. De ahí que las correlaciones mente-materia sean estructurales.

Según esta teoría, las correlaciones observables entre mente y materia son la consecuencia de la división epistémica de un campo psicofísico neutral. Dichas correlaciones no se deben a interacciones causales (causalidad eficiente), como suele hacer la ciencia cuando investiga la causalidad del cerebro hacia la mente, sino variaciones producidas por la ruptura de simetría ${ }^{3}$. Estas correlaciones fueron discutidas por Pauli y Jung en su correspondencia, examinada en Synchronicity (50), publicando finalmente sus ideas en una obra conjunta (51).

Desde el punto de vista psicológico, el proceso de medición se refiere a la transición del estado previo inconsciente al consciente, entendido como el paso de un campo no booleano a las alternativas binarias. Según Pauli y Jung, el paso del inconsciente al consciente es semejante a la transición entre las realidades mentales y materiales, de modo que el paso del inconsciente al consciente produce la localidad de los objetos. Meier (52) ha ampliado esta idea tratando problemas psicosomáticos desde esta perspectiva.

Tanto en Bohm y Hiley (42) como en el trabajo de Atmanspacher (38) se trata de una metafísica holística en la que lo mental y lo físico no son reducibles entre sí ni al campo neutro originario, sino que emergen de este rompiendo la simetría holística. Son teorías que exponen que, en el límite de una simetría universal que se rompe en el nivel psicofísico neutral, cada sistema tiene tanto un aspecto material como mental. Los actos inconscientes o protomentales, opuestos a los mentales, explicarían la diferencia. El caso de la mente humana dentro del campo mental es

\footnotetext{
3 "Según Pierre Curie, la ruptura de simetría tiene la siguiente función: para la aparición de un fenómeno en un medio, el grupo de simetría original del medio debe reducirse (romperse, en la terminología actual) al grupo de simetría del fenómeno (o a un subgrupo del grupo de simetría del fenómeno) por la acción de alguna causa. En este sentido, la ruptura de simetría es lo que 'crea el fenómeno'” (49).
} 
considerado de la misma importancia que su correlato material, el cerebro, dentro del campo material (53).

El único problema de esta concepción de la mente es que parece debilitar la concepción de realidad mayoritariamente aceptada social y científicamente. Lo que denominamos realidad no solo sería, como ya dijo Kant (3), una construcción subjetiva en el espacio-tiempo, sino que el propio espacio-tiempo sería una construcción mental emergente de un único campo subyacente.

\section{Conclusiones}

El precio de esta falta de fronteras entre sujeto-objeto sería la pérdida, en parte, del prestigio de la ciencia como conocimiento de la realidad, ya que habría que incluir el elemento subjetivo del observador. Eso no quiere decir que las denominadas ciencias duras sean inútiles o falsas, sino que habría que rebajar el concepto de verdad que actualmente posee: se trataría de incluir al observador en las observaciones o, aún más, habría que basarse en las relaciones entre sistemas (30). Pero aún más interesante podría ser el surgimiento de una nueva ciencia que unificase los ámbitos subjetivos y objetivos separados en la modernidad (mente-materia), lo que nos ayudaría a comprendernos a nosotros mismos.

La introducción del estudio de la mente en la ciencia (en física y biología, al menos) puede suponer el fin de muchas de las dicotomías planteadas al comienzo del pensamiento científico derivadas de la separación de mente y materia, como la división entre ciencias humanas y ciencias experimentales, lo social y lo biológico, o simplemente lo natural frente a lo artificial, ya que, si incorporamos la actividad mental dentro de la naturaleza, no habría por qué pensar en contrarios, sino solo distinguirlas en base a la percepción humana como adaptación necesaria para su supervivencia.

Frente a las limitaciones de la ontología clásica de un mundo mecánico material, la ontología de Bohm y Hiley (42) o la de Atmanspacher $(19,38)$ aportan una comprensión de la mente mucho más sencilla y comprensible que cualquier dualismo o el materialismo reductivo actual, siendo capaces de explicar la brecha entre mente y materia y resolviendo al mismo tiempo el problema abierto por Descartes de la relación mente-cuerpo. La información desempeña un papel fundamental como puente entre mente y materia.

Socialmente, una perspectiva holística también puede cambiar radicalmente la forma en que entendemos la relación entre el individuo y su entorno (social y físico). Mientras que en el mecanicismo se reduce a individuos que interactúan entre ellos, en una visión holística, cada individuo es parte de un todo que incluye la sociedad y el entorno físico, los cuales constituyen la información de la memoria del individuo. De modo que, probablemente, los contenidos mentales no sean produc- 
tos de un cerebro individual, sino manifestaciones del estado de los grupos sociales en los que el individuo participa. En esta perspectiva, el sufrimiento es universal y los esfuerzos en investigar, sanar o prevenir no deben dirigirse tanto a los individuos como a detectar las incoherencias a nivel social. En psiquiatría y en neurociencias, en general, supondría la aceptación de que el comportamiento no responde solo a la maquinaria cerebral, sino a factores de información (los que se denominan sociales o intersubjetivos), a menudo inconscientes, lo que podría aclarar tanto la etiología de los trastornos mentales como el propio papel de esta práctica médica, muy cuestionada por carencia de pruebas clínicas y tratamientos perjudiciales.

Entre las ventajas que pueden aportar estas ideas para un nuevo modelo de psiquiatría figuran las siguientes: es ciencia, no simples especulaciones; se puede someter a comprobación empírica; la teoría cuántica es más adecuada para entender la actividad mental que el mecanicismo, dada la complejidad y sutileza de los procesos mentales (se hace cargo de los no observables); suaviza las relaciones humanas al introducir el factor de indeterminación y, por tanto, indica la necesidad de flexibilidad; atenúa el estigma del error cognitivo sobre la percepción de la realidad (la realidad depende del observador); y elimina el dogmatismo, ya que la física cuántica nos recuerda que solo podemos predecir la naturaleza de un proceso a partir de la inferencia estadística, pero nunca determinarlo del todo, lo que es más adecuado en biología, en medicina y, sobre todo, en psiquiatría, disciplinas en las cuales es difícil predecir el resultado. Posiblemente un cambio de paradigma en nuestra comprensión de la realidad y de nosotros mismos también podría conducir a un cambio social transformando nuestras mentes newtonianas (así hemos sido formados en la escuela) individualistas en mentes más conscientes de nuestro entorno.

\section{Bibliografía}

(1) Hempel CG. Aspects of scientific explanation and other essays in the philosophy of science. New York: The Free Press, 1965.

(2) Aragona M. Neopositivism and the DSM psychiatric classification. An epistemological history. Part 1: Theoretical comparison. Hist Psychiatry. 2013; 24(2): 166-179.

(3) Kant I. Crítica de la razón pura. Buenos Aires: Ediciones Colihue SRL, 2009.

(4) Husserl E. On the phenomenology of the consciousness of internal time (18931917) (Vol. 4). Berlín: Springer Science \& Business Media, 2012.

(5) Heidegger M. Ser y tiempo. Santiago de Chile: Editorial universitaria, 2005.

(6) Merleau-Ponty M. Phénoménologie de la perception. París: Éditions Gallimard, 2013. 
(7) Varela FJ. Neurophenomenology: A methodological remedy for the hard problem. J Conscious Stud. 1996; 3(4): 330-349.

(8) Freeman WJ, Vitiello G. Nonlinear brain dynamics as macroscopic manifestation of underlying many-body field dynamics. Phys Life Rev. 2006; 3(2): 93-118.

(9) Parnas J, Sass LA, Zahavi D. Rediscovering psychopathology: the epistemology and phenomenology of the psychiatric object. Schizophr Bull. 2012; 39(2):270-277.

(10) Gallagher S. Mutual enlightenment: recent phenomenology in cognitive science. J Conscious Stud. 1997; 4(3): 195-214.

(11) Fuchs T. The challenge of neuroscience: psychiatry and phenomenology today. Psychopathology 2002; 35(6): 319-326.

(12) Bohm D. Wholeness and the implicate order (Vol. 10). Londres: Psychology Press, 2002.

(13) Hume D. Tratado de la naturaleza humana. Madrid: Orbis, 1984.

(14) Churchland PM. The evolving fortunes of eliminative materialism. En: McLaughlin B, Cohen J (eds). Contemporary debates in philosophy of mind. Oxford: Blackwe11, 2007.

(15) Dennett DC. Consciousness explained. Boston: Little Brown, 2017.

(16) Frankish K. Illusionism as a theory of consciousness. J Conscious Stud. 2016; 23(1112): 11-39.

(17) Szasz TS. The problem of psychiatric nosology: A contribution to a situational analysis of psychiatric operations. Am J Psychiatry 1957; 114(5): 405-413.

(18) Edelman GM, Tononi G. El universo de la conciencia: cómo la materia se convierte en imaginación. Barcelona: Crítica, 2002.

(19) Atmanspacher H. Quantum approaches to consciousness. En: Zalta EN. The Stanford Encyclopedia of Philosophy. 2015. [Internet] [Consultado en enero 2020]. Disponible en: https://plato.stanford.edu/archives/sum2015/entries/qt-consciousness/

(20) Bitbol M. Beyond panpsychism: the radicality of phenomenology. En: Menon S, Nagaraj N, Binoy VV (eds). Self, culture and consciousness. Singapur: Springer, 2017; pp. 337-356.

(21) Kim J. Psychophysical supervenience. Philosophical studies: An International Journal for Philosophy in the Analytic Tradition 1982; 41(1): 51-70.

(22) Chalmers DJ. Facing up to the problem of consciousness. J Conscious Stud. 1995; 2(3): 200-219.

(23) Cole D. The chinese room argument. En: Zalta EN. The Stanford Encyclopedia of Philosophy. 2015. [Internet] [Consultado en enero 2020]. Disponible en: https:// plato.stanford.edu/archives/spr2019/entries/chinese-room/

(24) Morris CW. Foundations of the theory of signs. (International encyclopedia of unified science; Foundations of the unity of science). Chicago: Chicago University Press, 1938; pp. 1-59.

(25) Mitra R, Sapolsky RM. Acute corticosterone treatment is sufficient to induce anxiety and amygdaloid dendritic hypertrophy. P Natl Acad Sci USA 2008; 105(14): 5573-5578.

(26) Rodrigues SM, LeDoux JE, Sapolsky RM. The influence of stress hormones on fear circuitry. Ann rev neurosci. 2009; 32: 289-313. 
(27) Ben-Shaanan TL, Schiller M, Azulay-Debby H, Korin B, Boshnak N, Koren T, et al. Modulation of anti-tumor immunity by the brain's reward system. Nat Commun. 2018; 9(1): 2723.

(28) Feigl H. The 'mental' and the 'physical'. Minnesota studies in the philosophy of science 1958; 2(2): 370-497.

(29) Wittgenstein L. Tractatus logico-philosophicus. Londres: Routledge, 2013.

(30) Rovelli C. Physics needs philosophy. Philosophy needs physics. Found Phys. 2018; 48(5): 481-491.

(31) Chalmers DJ. The conscious mind: in search of a fundamental theory. Oxford: Oxford University Press, 1996.

(32) Goff P. Consciousness and fundamental reality. Oxford: Oxford University Press, 2017.

(33) Globus G. A quantum monadological solution to the measurement, consciousness and qualia problems. NeuroQuantology 2014; 12(2).

(34) Mender D. The cunning of the observable: generalized Fourier duality, the hard problem, and natural kinds of psychopathology. Quantum Biosystems 2013; 5(2): 43-53.

(35) Spinoza B. Ética demostrada según el orden geométrico. Madrid: Editorial Trotta, 2000.

(36) Damasio AR. En busca de Spinoza: neurobiología de la emoción y los sentimientos. Barcelona: Grupo Planeta, 2005.

(37) Tononi G, Koch C. The neural correlates of consciousness. Ann NY Acad Sci. 2008; 1124(1): 239-261.

(38) Atmanspacher H. Levels of unconsciousness and their formal structure. J Anal Psychol 2014; 59(3): 385-390.

(39) Bohr N. The quantum postulate and the recent development of atomic theory (Vol. 3). Edinburgo: R. \& R. Clarke Ltd, 1928.

(40) James W. The principles of psychology. Redditch, Worcestershire: Read Books Ltd, 2013.

(41) Bohm D. Wholeness and the implicate order (Vol. 10). Londres: Psychology Press, 2002.

(42) Bohm D, Hiley BJ. The undivided universe: An ontological interpretation of quantum theory. Londres: Routledge, 2006.

(43) Pribram KH (ed.). Rethinking neural networks: Quantum fields and biological data. Londres: Psychology Press, 2014. (44) Bell JS. Einstein-Podolsky-Rosen experiments. En: Bell M, Gottfried K, Veltman M. John S Bell on the foundations of quantum mechanics. Singapur: World Scientific Publishing Company, 2001; pp. 74-83.

(45) Busemeyer JR, Wang Z, Townsend JT. Quantum dynamics of human decision-making. J Math Psychol. 2006; 50(3): 220-241.

(46) Atmanspacher H, Filk T. The Necker-Zeno model for bistable perception. Top cogn Sci. 2013; 5(4): 800-817.

(47) Atmanspacher H, Filk T, Foundation P. Complexity and non-commutativity of learning operations on graphs. BioSystems 2006; 85(1): 84-93.

(48) Gabora L, Aerts D. Contextualizing concepts using a mathematical generalization of the quantum formalism. J Exp Theor Artif In. 2002; 14(4): 327-358.

(49) Brading K, Castellani E, Teh N. Symmetry and symmetry breaking. The Stanford 
Encyclopaedia of Philosophy (Winter 2017 Edition) Zalta EN (ed.). Disponible en: https://plato.stanford.edu/entries/symmetry-breaking/

(50) Combs A, Holland M. Synchronicity: Through the eyes of science, myth, and the trickster. New York: Paragon House Publishers, 1990.

(51) Jung CG, Pauli W. Naturerklärung und Psyche: Synchronizität als ein Prinzip akausaler Zusammenhänge. Zürich: Rascher, 1952.

(52) Meier CA. Psychosomatik in Jungscher Sicht. Psyche 1962; 15(10): 625-38.

(53) Skrbina D. Panpsychism as an underlying theme in western philosophy. A survey paper. J Conscious Stud. 2003; 10(3): 4-46. 